\title{
Load Balancing in Wireless Networks using SDN-enabled Infrastructure: Traffic Analysis
}

\author{
Heng Meng Heang \\ School of Computer Science and \\ Technology, Chongqing University of \\ Posts and Telecommunications, \\ Chongqing,400065 \\ China \\ hengmengheang@gmail.com
}

\author{
Syed Mushhad M. Gilani \\ School of Computer Science and \\ Technology, Chongqing University of \\ Posts and Telecommunications, \\ Chongqing, 400065 \\ China \\ mushhad@uaar.edu.pk
}

\author{
Hemn B. Abdalla \\ School of Communication and \\ Information Engineering, Chongqing \\ University of Posts and \\ Telecommunications, \\ Chongqing, 400065 \\ China \\ hemn.db85@yahoo.com
}

\author{
Tang Hong \\ School of Communication and \\ Information Engineering, Chongqing \\ University of Posts and \\ Telecommunications, \\ Chongqing, 400065 \\ China \\ tanghong@cqupt.edu.cn
}

\begin{abstract}
The current wireless network has huge problems to deliver the good throughput at minimum packet dropped ratio. The one reason is a lack of resource management which leads to load imbalanced environment. Besides, the typical WLAN architectures are based on proprietary hardware that does not permit to deploy the innovative technologies and network services. Software-Defined Network (SDN) familiarizes as future Internet technology that brings invention in the wireless network paradigm. In order to resolve the load balancing issue, we proposed the SDN-based WLAN environment which contains the load balancer to take a view of each of the resources of WLAN as well as distribute them. It permits the dynamic association and re-association of WLAN clients among the adjacent access points (APs) in each possible situation. The results of simulation demonstrate that the SDN-based WLAN environment ensures that traffic load will not exceed for a long time among adjacent APs, and improve the throughput of associated stations.
\end{abstract}

\section{CCS CONCEPTS}

Networks $\rightarrow$ Network performance evaluation; Network $p$ erformance analysis

Permission to make digital or hard copies of all or part of this work for personal or classroom use is granted without fee provided that copies are not made or distributed for profit or commercial advantage and that copies bear this notice and the full citation on the first page. To copy otherwise, to republish, to post on servers or to redistribute to lists, requires prior specific permission and/or a fee.

MOBIMEDIA 2017, July 13-14, Chongqing, People's Republic of China Copyright ( 2017 EAI 978-1-63190-156-0

\section{KEYWORDS}

Software-Defined Network, Throughput analysis, WLAN.

\section{INTRODUCTION}

IEEE introduced first WLAN standard in 1997, afterward, the access point (AP) journey started at a low data rate with limited range. The proliferation of mobile applications and data traffic continuously motivates for enlightening and introducing the new standards in WLAN. Subsequently, IEEE has done much work for improving the WLAN standards [12]. However, the load balancing in the existing wireless LANs is a key challenge, because IEEE 802.11 does not provide the automotive load balancing in WLAN environment [10]. The term load defines in IEEE 802.11 as the number of active process per AP, and the load balancing approach can distribute the equal number of the active process among them.

In the current IEEE 802.11 WLAN setup, APs are installed in the several localities. The user can receive multiple probes for association from one locality. Afterward, the user could associates with that AP which has excellent signal to noise ratio (SNR) or good received signal strength indicator (RSSI). In this situation, the user might be associated with the heavy traffic loaded AP and does not explore the potential of others available APs. Therefore, it causes the throughput degradation and loads imbalance in WLAN. Figure 1 depicts that Sta8 moves among two APs but do not have QOS measure to help it for the association. Therefore, the load imbalanced environment creates problems that lead to offensive response times and imbalanced bandwidth assignment and extensive waiting time in WLANs. 
Software-Defined Network (SDN) introduces the logical separation between data plane and control plane [8]. The concept of SDN has been successfully implemented in the wired networks i.e. data centers, LAN switches and cloud networks [2] The popularity of SDN attracts the benchmark traders and service providers to adopt the concept of SDN in wireless networks [1]. With the increasing demand for smart applications and massive growth in mobile devices, it is necessitated to adopt the SDN technology paradigm in WLAN.

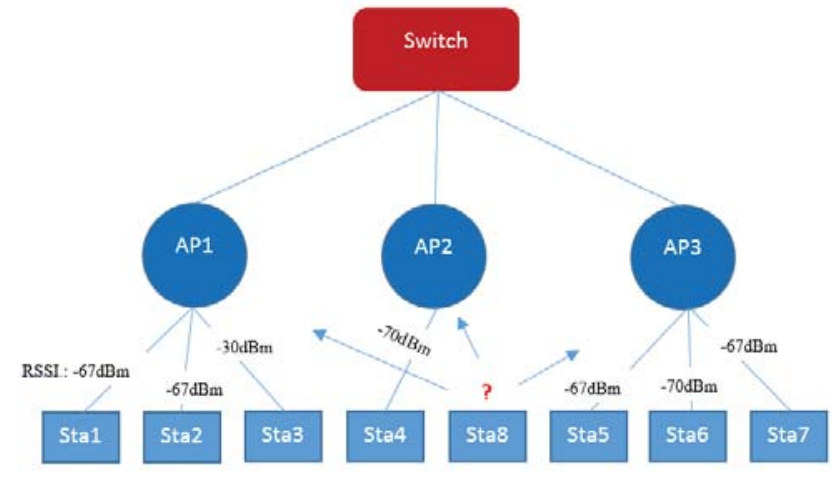

Figure 1. Station Association Problem in WLAN

The main contribution of this research work is to design the load balancing environment that provides the load association control under the umbrella of SDN. The performance evaluation of the proposed approach has performed through simulation. We decided to use Mininet [5] to develop the SDN-based WLAN infrastructure. It provides the accurate model of shared wireless channel and efficiently simulates the network for a variety of topologies with the user defined parameters. Other key contributions of this paper are summarized as follows:

- We figured out the shortcomings of traditional WLAN through the implementation of multiple applications using UDP and TCP protocols to investigate the performance of WLAN clients.

- We designed the SDN-based WLAN environment that includes the load balancer which enables the execution of load balancing procedure for WLAN.

- We further build the simulation topologies to analyze the proposed approach's effects and also perform the comparison with the traditional system.

The rest of this paper is organized as follows: Section 2 presents the issues of traditional WLAN and motivations towards SDN. Moreover, we talked about Open Flow, Flow Tables, messages of Open Flow, Use cases of SDN in the wireless networks and technical background. The load balancing approach that included load-aware clients association is presented in Section 3. In section 4, we presented the simulation, and evaluation is done in both quantitative and qualitative perspective. The methods of evaluating the proposed approach and experiments performed are discussed, along with the corresponding results. Finally, section 5 contains the concluding statements and points out some possible future directions.

\section{BACKGROUND}

\subsection{Traditional WLAN}

APs provide a virtual connection to the user from any place that falls in the range of wireless signals. Mostly vendor (CISCO Meraki, HP) introduces two different varieties of APs; Indoor AP and Outdoor AP [4]. Indoor APs based on low profile industry design with limited signal range and can be affected by external interference. Although Outdoor APs have high capacity, dual radio frequency, enterprise security, client session handler and other related modules. Two different frequency bands can activate in the same locality without signal interference. Dualband APs have the ability to support $2.4 \mathrm{GHz}$ card for connecting low data rate applications, and $5 \mathrm{GHz}$ card can support the high bandwidth required applications.

\subsection{Shortcomings of Traditional WLAN}

Regrettably, the traditional WLAN could be less capable of incorporating new technologies and facing several challenges: a) Poor resource allocation. b) Lack of administration activities. c) Inter-communication gap between APs. d) It supports only the fixed network characteristics and does not permit to share the single AP [3] across different ISPs. e) Reconfiguration of the APs [7] is a challenge after the deployment. f) Massive packet drop during the realization method in different network domains [7]. g) The poor channel allocation, deep coverage and unsatisfied user experience due to the sharing same airtime of traditional APs [11].

\subsection{Motivation towards Non-traditional WLAN}

Above mentioned obstacles motivate researchers and experts to figure out a possible solution for boosting the performance of WLANs to support the future Internet applications. Most of the technologies inherit from the local area network (LAN) infrastructure to enhance the wireless network features that can fulfill the future Internet desires. Such as distribution of various network characteristics for the diverse nature of services through the same AP can improve the QoS and QoE.

\subsection{SDN outcomes in WLAN}

SDN persuade network operators and vendors due to its programmable interface, ability to work in a heterogeneous network by quick scheduling, reduce cost through on-demand resource utilization and customized the new technologies beyond the open APIs [1] [6]. SDN introduces an abstraction of the forwarding and decoupling of the data plane from control plane as illustrated in Figure 2, which gives freedom to deploy the innovative applications and network services. The Ethane was one of the initial projects of SDN that introduced for enterprise network and implemented using customized packages i.e. OpenWrt, NetFPGA and open source operating system. Google is presented one more practical example of a real application of the SDN in the context of data centers in early 2012. The first SDN project in the wireless network was 
OpenRoads [9] that enables the seamless mobility across different wireless infrastructures.
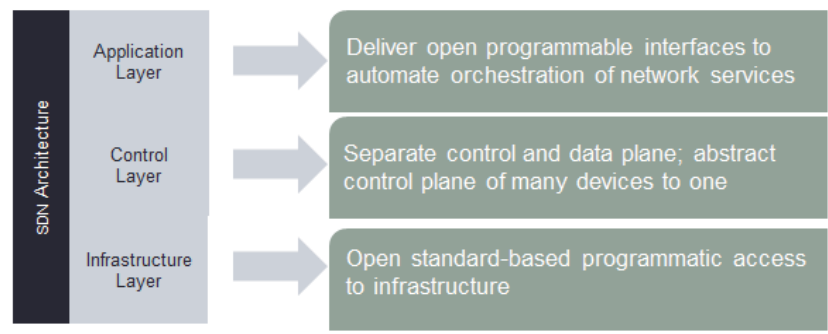

Figure 2. SDN Reference Architecture

\section{PROPOSED ENVIRONMENT}

The SDN-based WLAN environment is developed to implement the proposed approach. As illustrated in Figure 3, the load balancer module is installed at the top of the SDN controller. The controller is notified to the load balancer whenever new association attempts occur or find multiple data transmission paths. The load balancer activates the load calculation module to find the least loaded AP and send a response to the controller for further action. The client association procedure is shown in Figure 4.

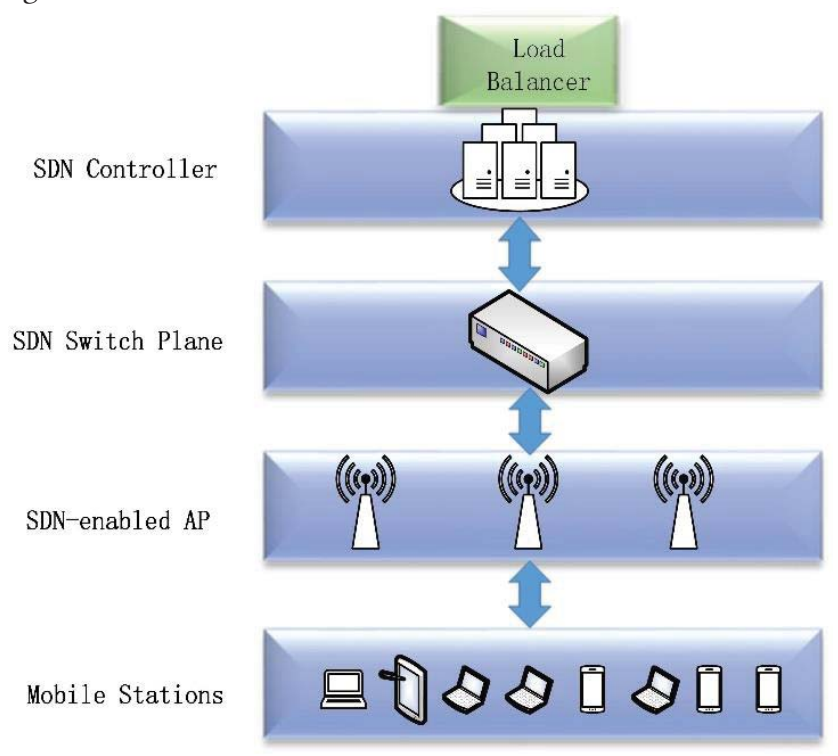

Figure 3. An illustration of SDN-enabled WLAN environment

The SDN controller is an important entity which can provides the global information of the entire network. It also optimizes the resource allocation on the basis of real-time network information that helps to the load balancer for the implementation of traffic rules and algorithms. The SDN controller receives new station probe request, it defines the traffic path as per defined rules and sends an acknowledgment in response (Figure 4). The POX controller handles the real-time information processing task. As a central network entity, it is mainly responsible for user's traffic management and enables the communication among the internetworking devices. The controller is also able to deny the association or redirect it to other APs using the load balancer application-defined rules.

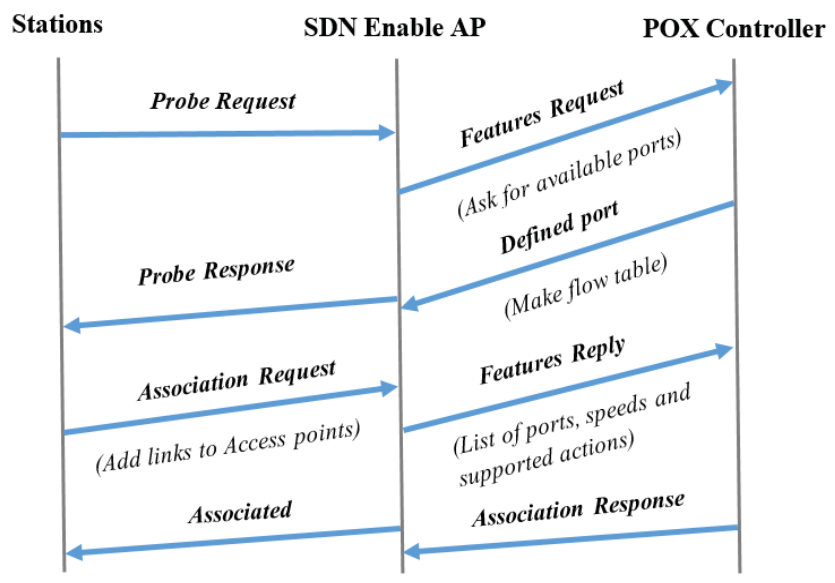

Figure 4. Messages Sequence for Station-AP-Controller

SDN switch enables the OpenFlow protocol to keep the information into flow tables which are utilized for the packet matching and forwarding. Each switch sends their information to the load balancer through the SDN controller.

In order to achieve the load balancing in WLAN, it is necessary to synchronize the current network information of adjacent APs. The information includes the associated number of stations, the capacity of AP, current workload of AP and the distance between nearby APs and associated stations. The ultimate goal is to find the load path towards best-AP for data transmission. Each AP configures with OpenFlow protocol and acts as an agent of SDN controller. In the proposed WLAN setup, whenever an AP receives probe request, the POX controller acquires the current information of AP along with traffic path directions and ports. Afterward, it forwards the information to the load balancer for load comparison among nearby APs and finally decides the transmission path towards least loaded AP. Function handles the initial station-AP association is shown in Figure 5.

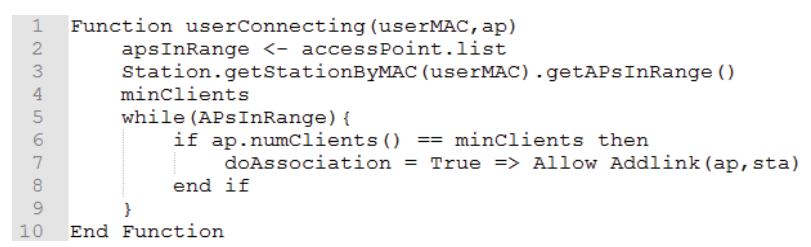

Figure 5. Client association control function

\section{SIMULATION AND EVALUATION}

The goal of this research work is to observe the reported load traffic, bandwidth, and packet loss. The opportunity of implementing and testing new methods in a real testbed is problematic and expensive. The network simulator/emulator 
gives a platform to testify the idea before the implementation of actual infrastructures. However, the available network simulators are expensive and limited support to OpenFlowenabled wireless networks. In order to simulate the wireless network scenario with SDN, we use Mininet version 2.2.1 [5] that runs in Ubuntu 14.04, which is developed by Stanford University and released under a permissive BSD Open Source license. An additional benefit is that the python code used to program in the simulator can be deployed into a real testbed. The next subsection explains the steps needed in order to set up the experimental environment.

\subsection{Simulation Setup}

In this research, we simulate the multiple applications with different protocols i.e. TCP, UDP and FTP for testing the load balancing association control in WLAN. The simulation setup setting is given in Table 1. The Pox Controller is installed as a controller of the network. The supporting software tools are a virtual box that runs the virtual network and Eclipse as IDE.

Table 1. Simulation setup settings

\begin{tabular}{ll}
\hline Operating System & Ubuntu 14.04 \\
\hline Controller & POX \\
CPU & Intel ${ }^{*}$ Xeon ${ }^{\circledR E} 5-26202.00$ \\
& $\mathrm{GHz}^{*} 4$ \\
Memory & $4 \mathrm{~GB}$ \\
VGA & NVIDIA GeForce 9300 \\
Link rate Range & Min $5 \mathrm{Mbit} / \mathrm{s}$ \\
& Max $50 \mathrm{Mbit} / \mathrm{s}$ \\
Packet rate Range & Min 01 packets/s \\
& Max 10 packets/s \\
Packet Size Range & Min $5.00 \mathrm{Mb}$ \\
& Max $35.00 \mathrm{Mb}$ \\
\hline
\end{tabular}

We simulate an IEEE 802.11g network, operating in the infrastructure mode. The MAC layer is configured to work with the RTS-CTS exchange for every frame transmission. The detail of WLAN parameters are given in Table 2.

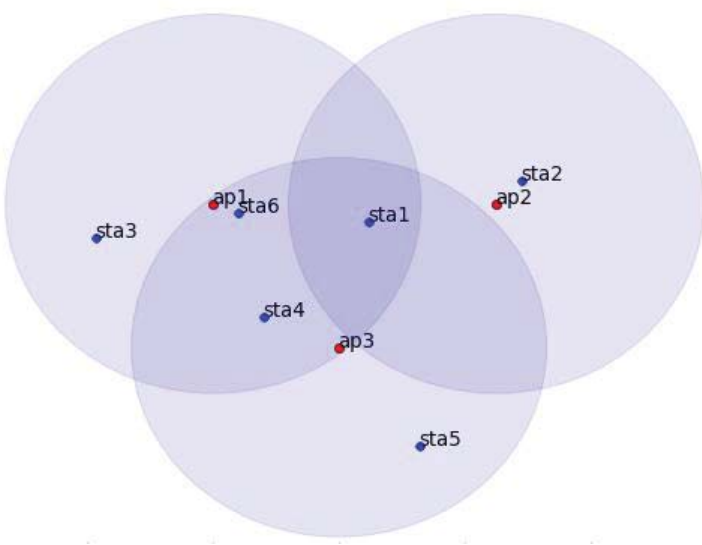

Figure 6. An example of Mininet simulation
Table 1. WLAN Parameters Settings

\begin{tabular}{ll}
\hline AP & $802.11 \mathrm{~g}\{\mathrm{AP} 1, \mathrm{AP} 2, \mathrm{AP}\}$ \\
\hline SSID & $\{$ new-ssid1, new-ssid2, new-ssid3 $\}$ \\
Tx-Power & $\{20 \mathrm{dBm}, 20 \mathrm{dBm}, 20 \mathrm{dBm}\}$ \\
Frequency & $\{2.412 \mathrm{GHz}, 2.417 \mathrm{GHz}, 2.422 \mathrm{GHz}\}$ \\
Channel & Channel $6\{20 \mathrm{MHz}, 20 \mathrm{MHz}, 20 \mathrm{MHz}\}$ \\
Data-Rate & $\{54.0 \mathrm{Mbit} / \mathrm{s}, 54.0 \mathrm{Mbit} / \mathrm{s}, 54.0 \mathrm{Mbit} / \mathrm{s}\}$ \\
Stations & \multicolumn{1}{c}{40} \\
\hline
\end{tabular}

\subsection{Performance Evaluation}

In the first test evaluation, we build a simulation topology to analyze the TCP traffic that includes 30 stations which are randomly associated with 3 APs. In traditional WLAN setup, 5 stations are associated with AP1, 10 stations are associated with AP2 and 15 stations are established a connection with AP 3, on the basis of RSSI value.

Figure 7 depicts the results of TCP traffic in the traditional WLAN, in which the connected stations to AP3 receives less throughput as compared to the stations of AP1 and AP2. It can be seen that this situation makes the overall network load imbalance and also can choke the network if more stations connect with AP3. Next, we run the simulation in SDN-based WLAN in which load balancer equally distribute the number of stations among adjacent APs that receives good throughput. However, in a real WLAN setup, the number of associated stations may not be equal. Note that, in the proposed environment, the throughput of stations connected with AP3 is increased $10 \mathrm{Mbps}$ averagely.

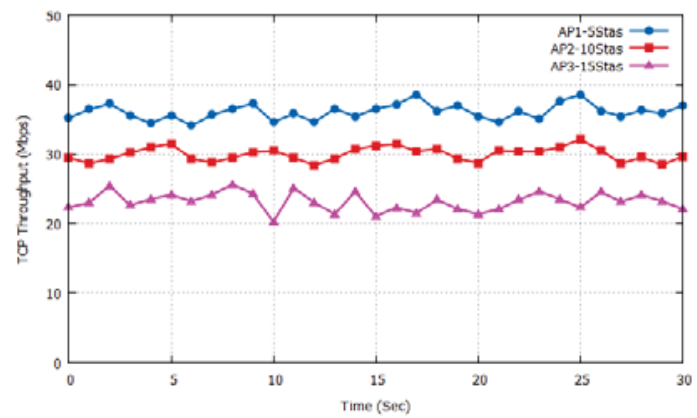

(a). TCP Traffic in Traditional WLAN

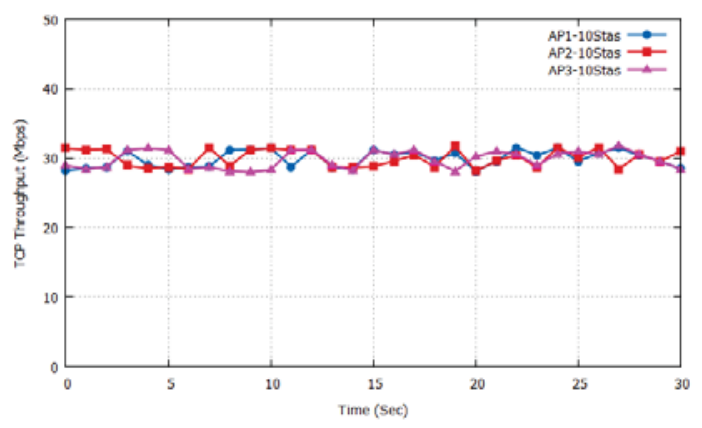

(b). TCP Traffic in SDN-based WLAN 


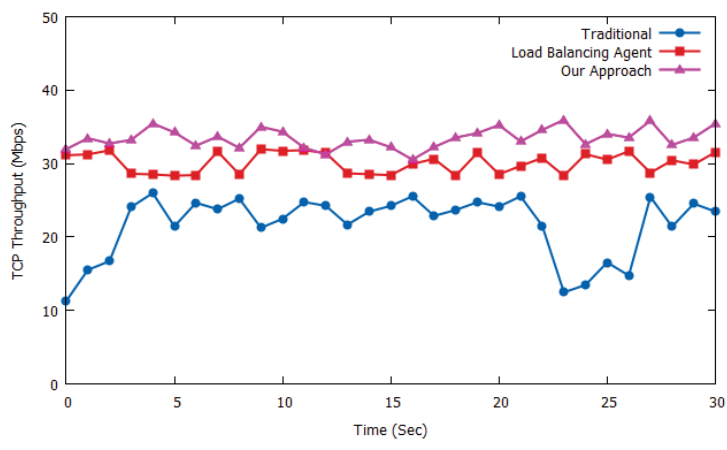

(c). Throughput Comparison

Figure 7. Results of TCP Traffic

For the second test evaluation, we configured web hosting server (Figure 8) to examine the FTP uplink and downlink traffic. We placed the file $($ size $=320.2 \mathrm{Mb})$ at a web server that is available for the downloading and also permits the associated stations for uploading files.

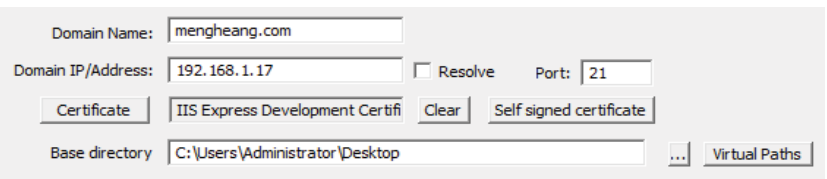

Figure 8. Screenshot of configuration IIS web server

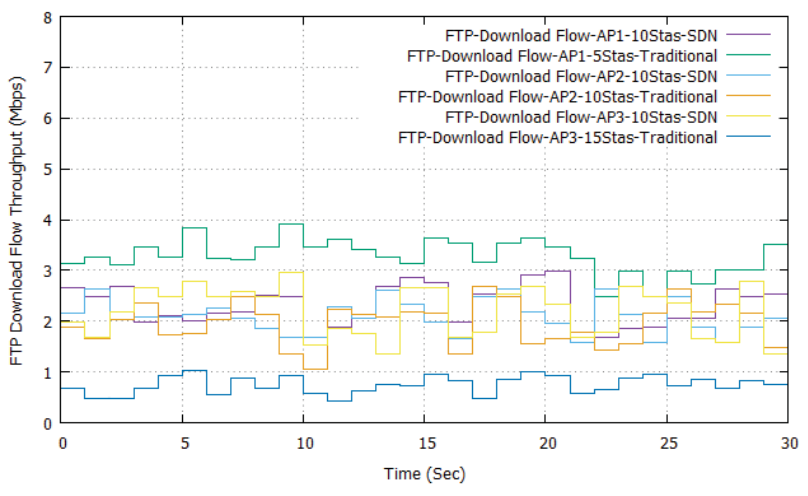

(a) FTP Download Traffic Comparison

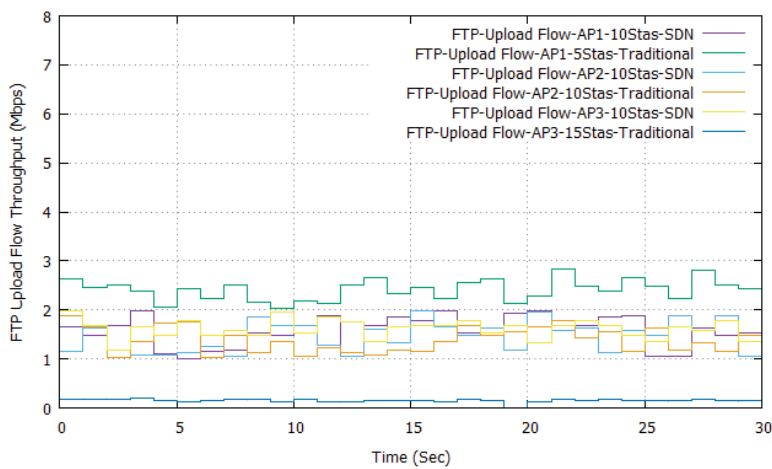

(b) FTP Upload Traffic Comparison Figure 9. Results of FTP Traffic Investigation
First, we execute the experiment in the traditional environment, in which AP1, AP2, and AP3 are randomly connected with 5, 10, and 15 stations, respectively. The second experiment is performed in the proposed environment that distributes the number of stations among adjacent APs and makes the overall balanced environment. The associated stations were started the file downloading process at time $\mathrm{t}=0$, as shown in Figure 9 (a). We measured the average download traffic i.e. AP1-10Stas-SDN receives 1.69Mbps, AP1-5Stas-traditional receives 2.01Mbps, AP2-10Stas-SDN receives $1.57 \mathrm{Mbps}$, AP2-10Stas-traditional receives $1.492 \mathrm{Mbps}$, AP3-10Stas-SDN receives $1.63 \mathrm{Mbps}$, and AP3-15Stas-traditional receives $0.59 \mathrm{Mbps}$. Note that, in the proposed environment the average download traffic is improved and balanced among adjacent APs.

The average FTP upload traffic flow is observed i.e. AP1-10StasSDN is $0.91 \mathrm{Mbps}$, AP1-5Stas-Traditional is $1.15 \mathrm{Mbps}$, AP210 Stas-SDN is $0.90 \mathrm{Mbps}$, AP2-10Stas-Traditional is $0.872 \mathrm{Mbps}$, AP3-10Stas-SDN is $0.914 \mathrm{Mbps}$, and AP3-15Stas-Traditional are $0.11 \mathrm{Mbps}$. It is noted that when the WLAN is not balanced, then the average upload traffic is less as a comparison with the proposed environment.

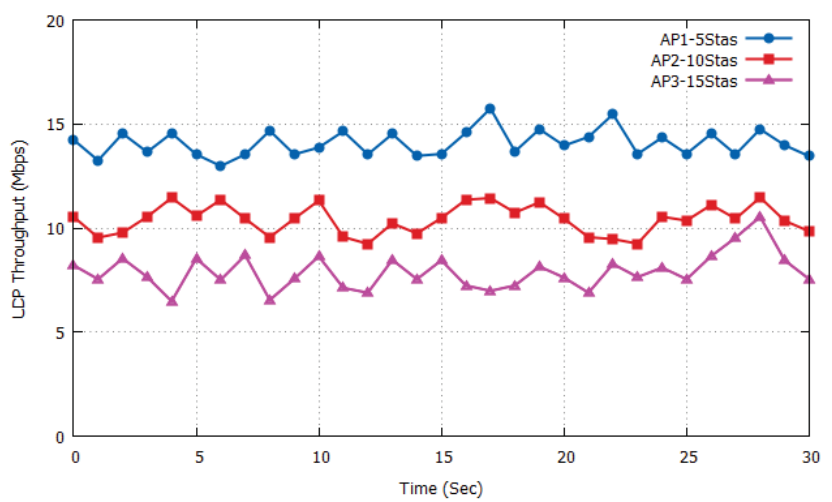

(a) UDP Traffic in Traditional WLAN

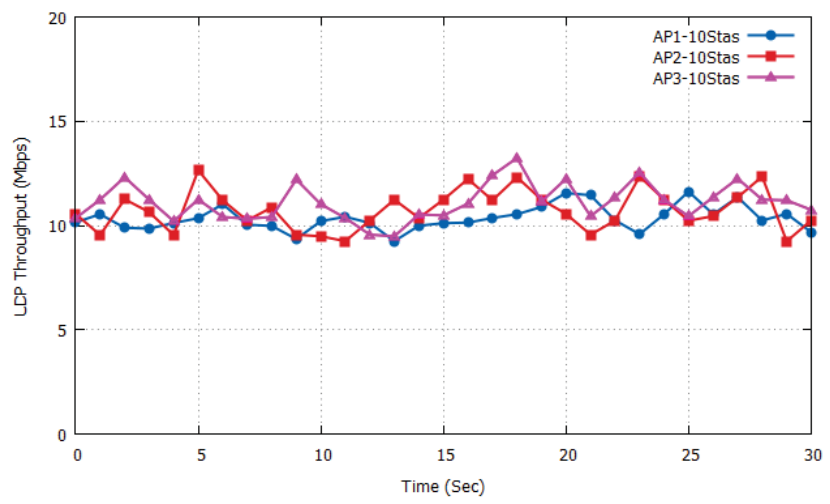

(b). UDP Traffic in SDN-based WLAN Figure 10. Results of UDP Traffic Comparison

Figure 10 plots the outcome of a UDP-based experiment that was performed in the traditional and proposed environment. As, it can be seen in Figure 10(a), the stations are connected with AP3 
receives the low throughput i.e. AP3 with 15 Stas receives 7.89Mbps. After the deployment of proposed approach the numbers of stations are equally distributed among nearby APs. The average UDP throughput of AP1 with 10 Stas is $10.34 \mathrm{Mbps}$, AP2 with 10 Stas is $10.73 \mathrm{Mbps}$, and AP3 with10 Stas is $11.1 \mathrm{Mbps}$, as illustrated in Figure 10(b).

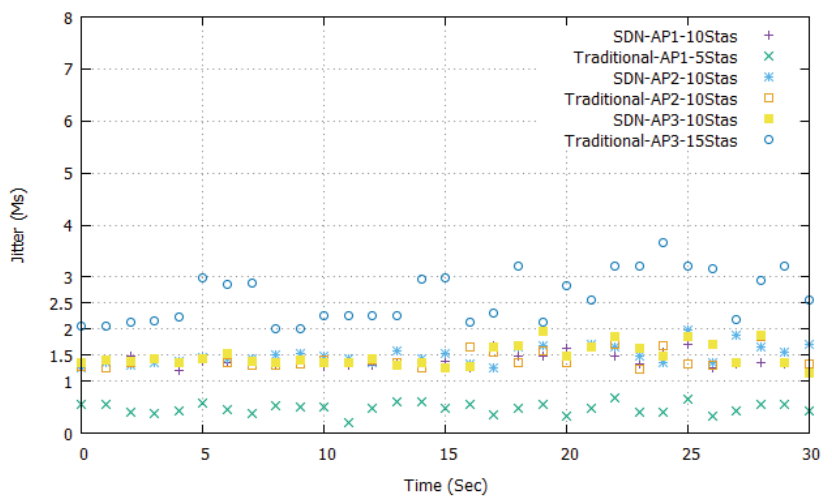

Figure 11. Marks of Jitter in the Traditional and the SDNbased WLAN

Figure 11 plots the marks of Jitter during the UDP transmission in the both environment, traditional and SDN. The UDP delay comprises on the medium access delays at the data link layer when MAC address of source and destination is not coordinated. First, we investigate the Jitter values in the traditional WLAN environment. During the UDP transmission 5 Stas which are associated with traditional-AP1 receives $0.47 \mathrm{~ms}$ average Jitter values, the associated Stas of AP2 and AP3 receives average Jitter values are $1.5 \mathrm{~ms}$ and $2.16 \mathrm{~ms}$, respectively. The second experiment is performed in the SDN-based WLAN with UDP protocol to observe the Jitter values, i.e. the associated Stas of AP1, AP2 and AP3 receive average Jitter values are $1.39 \mathrm{~ms}, 1.41 \mathrm{~ms}$, and $1.48 \mathrm{~ms}$, respectively. As it can be seen, the Jitter values are significantly reduced as compared with the traditional approach.

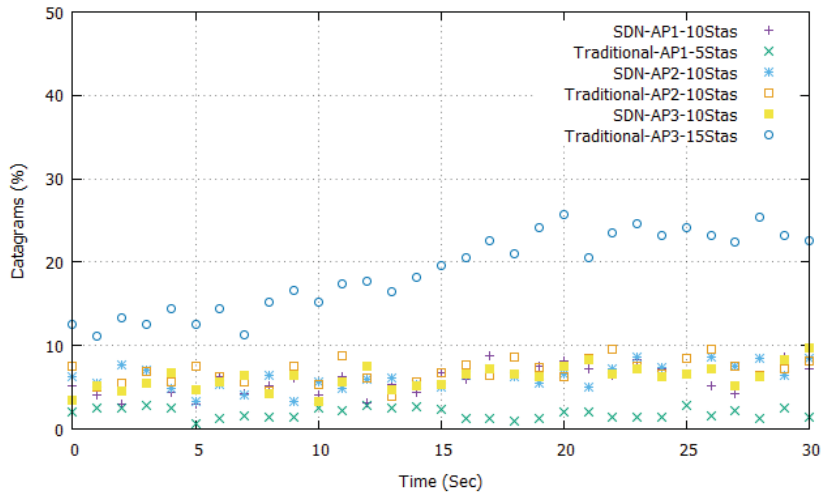

Figure 12. Datagram Dropped Ratio Comparison

In Figure 12, first, we study the impact of datagram dropped during the UDP traffic communication in the traditional WLAN. The traditional-AP1 is associated with 5 Stas, and the average datagram dropped ratio of Stas is $1.88 \%$. The traditional-AP2 is associated with 10 Stas, and average datagram dropped ratio is
6.9\%. The traditional-AP3 is associated with 15 Stas, and the average datagram dropped ratio is $18.87 \%$. The same experiment is performed with the SDN-enabled APs to observed the datagram dropped percentage i.e. 10 Stas are associated with SDN-AP1, and the average packet dropped ratio of Stas is $5.83 \%$. The SDN-AP2 is associated with 10 Stas, and average packet dropped ratio is $6.21 \%$ that is less than traditional AP1. SDN-AP3 is associated with 10 Stas with average datagram dropped ratio $6.12 \%$ that is significantly lower than traditional AP3. As it can see (Figure 12), in the proposed environment, the average datagram dropped ratio is comparatively controlled.

\section{Conclusion}

In this paper, we proposed the load balancing environment to enhance the throughput of the associated stations. The proposed environment is based on the SDN technology paradigm. With the advent of SDN technology, the proposed load balancing algorithm dynamically selects the lowest-loaded AP from the sharing locality for the client association. Our solution does not require any changes to the client side which ensure the implementation in the real world. The obtained results ensure the smooth throughput of associated stations and a decrease in packet drop and Jitter percentage. In the proposed environment, the load balancer provides access to service operators through the northbound interface for the further implementation of innovative ideas and algorithms. In the future work, we will testify the proposed environment in a dense wireless network with the support of multimedia contents.

\section{ACKNOWLEDGMENTS}

This work was supported by Prospective Research Project on National Nature Science Foundation (61402065, 61501075), Future Networks (BY2013095-2-03) fund by Jiangsu Future Networks Innovation Institute.

\section{REFERENCES}

[1] Akyildiz, I.F., Lin, S.-C. and Wang, P. 2015. Wireless software-defined networks (W-SDNs) and network function virtualization (NFV) for $5 \mathrm{G}$ cellular systems: An overview and qualitative evaluation. Computer Networks. 93, (Dec. 2015), 66-79.

[2] Banikazemi, M., Olshefski, D., Shaikh, A., Tracey, J. and Wang, G. 2013. Meridian: an SDN platform for cloud network services. Communications Magazine, IEEE. 51, 2 (2013), 120-127.

[3] Bhanage, G., Vete, D., Seskar, I. and Raychaudhuri, D. 2010. SplitAP: leveraging wireless network virtualization for flexible sharing of WLANs. (2010), 1-6.

[4] Cisco Meraki: https://meraki.cisco.com/. Accessed: 2017-04-08.

[5] Fontes, R.R., Afzal, S., Brito, S.H., Santos, M.A. and Rothenberg, C.E. 2015 Mininet-WiFi: Emulating software-defined wireless networks. Network and Service Management (CNSM), 2015 11th International Conference on (2015), 384-389.

[6] Gilani, S.M.M., Hong, T. and Zhao, G. 2015. SN-FMIA: SDN and NFV enabled future mobile internet architecture. Advanced Communication Technology (ICACT), 2015 17th International Conference on (2015), 341-346.

[7] Hamaguchi, T., Komata, T., Nagai, T. and Shigeno, H. 2010. A Framework of Better Deployment for WLAN Access Point Using Virtualization Technique. (2010), 968-973.

[8] Sezer, S., Scott-Hayward, S., Chouhan, P.-K., Fraser, B., Lake, D., Finnegan, J., Viljoen, N., Miller, M. and Rao, N. 2013. Are we ready for SDN? Implementation challenges for software-defined networks. Communications Magazine, IEEE. 51, 7 (2013), 36-43.

[9] Yap, K.-K., Kobayashi, M., Underhill, D., Seetharaman, S., Kazemian, P. and McKeown, N. 2009. The stanford openroads deployment. Proceedings of the 4th ACM international workshop on Experimental evaluation and characterization 
(2009), 59-66.

[10] Yen, L.-H., Yeh, T.-T. and Chi, K.-H. 2009. Load balancing in IEEE 802.11 networks. Internet Computing, IEEE. 13, 1 (2009), 56-64.

[11] Yiakoumis, Y., Bansal, M., Covington, A., van Reijendam, J., Katti, S. and McKeown, N. 2014. BeHop: a testbed for dense WiFi networks. (2014), 1-8.

[12] IEEE 802 LAN/MAN Standards Committee(2015,)IEEE 802.11 "Working group timelines”. http://www.ieee802.org/11/Reports/802.11 Timelines.htm.

Accessed July 2015. 